\title{
Developing community-based urine sampling methods to facilitate dietary exposure biomarker technology for population assessment
}

\author{
T. Wilson ${ }^{1}$, N.D. Willis ${ }^{2}$, H. Zubair ${ }^{1}$, M. Beckmann ${ }^{1}$, L. Xie ${ }^{2}$, K. Tailliart ${ }^{1}$, A.J. Lloyd ${ }^{1}$, \\ J.C. Mathers ${ }^{2}$ and J. Draper ${ }^{1}$ \\ ${ }^{1}$ Institute of Biological, Environmental and Rural Sciences, Aberystwyth University, Aberystwyth SY23 3DA and \\ ${ }^{2}$ Human Nutrition Research Centre, Institute of Cellular Medicine, Newcastle University, Newcastle-upon-Tyne, NE4 \\ $5 P L, U K$
}

Dietary exposure and nutritional status have a huge impact on the health and well-being of individuals and of populations ${ }^{(1)}$ and it is widely accepted that there is great potential to use dietary advice/interventions to delay the development of age-related ill-health ${ }^{(2)}$. However, it is difficult to obtain accurate and reliable dietary exposure information from individuals using current tools (e.g. Food Frequency Questionnaires, diet diaries) because of bias and mis-reporting and this hinders research efforts to link specific foods/ dietary patterns to health-related phenotypes. The application of food intake biomarkers in readily-obtained biological samples would mitigate this problem ${ }^{(3)}$. In epidemiological studies, spot urines represent a relatively non-invasive sample for participants to collect in their home-settings. As well as preserving the compositional integrity of the sample, it is essential that any methodology has minimal impact on the day-to-day activities of participants. Importantly, to facilitate wide adoption, any method must also be inexpensive, require no specialised equipment and samples must be simple to store and transport.

Our initial studies using well established methods ${ }^{(3)}$ found spot urine collected, either just before bedtime or as a first morning void, had as much value for dietary exposure assessment as urines collected over a $24 \mathrm{hr}$ period. We thus explored the utility of a range of spot urine sampling protocols, assessing both acceptability and performance of different collection devices, storage and transport solutions. Urine samples were examined for stability using triple quadrupole mass spectrometry (QQQ-MS) methods able to quantify concurrently dietary exposure biomarkers of up to 20 foods of high public health importance in the UK.

Compliance of $100 \%$ was achieved by adult participants $(n=19$, without supervision) in collection of spot urines using the prototype home urine sampling kit developed during this project. An online questionnaire (participants, $n=31$ ) showed that the acceptability of home urine collection and storage using this bespoke method not different from that for existing methods using Universal tubes or a commercial device (Fig 1).
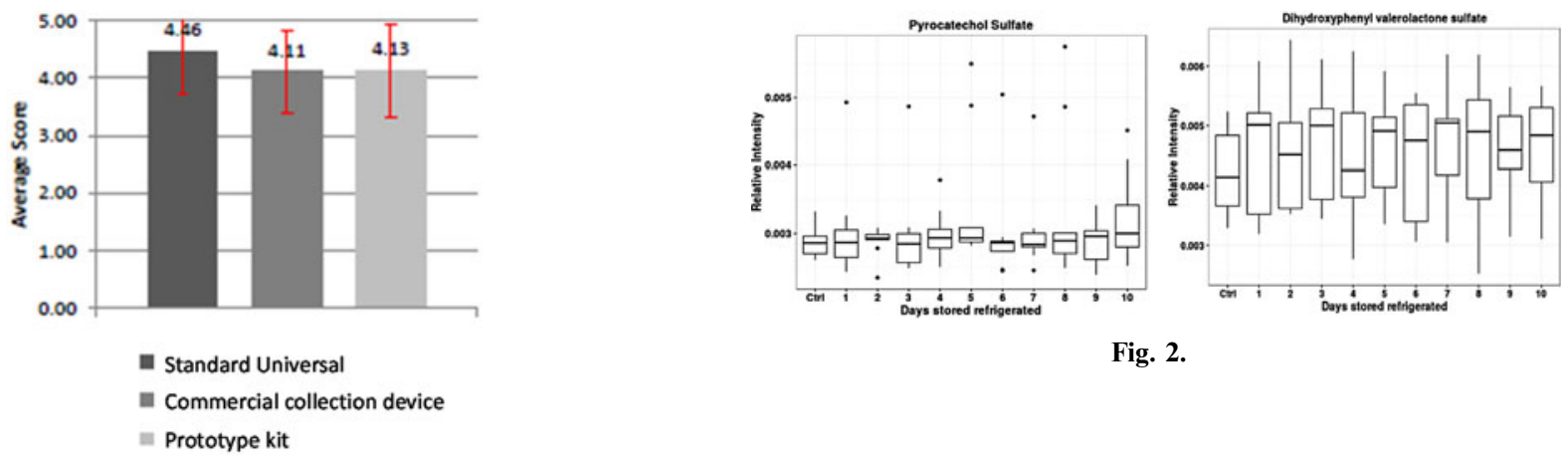

Fig. 2.

Fig. 1.

Metabolite signals representing all 18 dietary exposure biomarkers targeted for quantification showed no signs of degradation after prolonged storage (up to 10 days) at $+4^{\circ} \mathrm{C}$; data for two relatively labile fruit and vegetable exposure biomarkers are shown in Fig 2 . Additionally, the majority of metabolite signals remained stable after storage at ambient room temperature for 5 days or during transport between laboratories by the UK postal system.

In summary, self-collected spot urine samples appear suitable for development for routine use as a cost-effective approach for assessing dietary exposure in the community and this will facilitate monitoring of dietary behaviours in large-scale surveys and intervention studies.

1 GBD 2013 Risk Factors Collaborators (2015) Lancet 386, 2287-2323.

2 Celis-Morales C et al. (2015) Proc Nutr Soc. 74, 130-138.

3 Beckmann M et al. (2013) Proc Nutr Soc. 72, 352-361. 